\title{
Airport screening for Ebola
}

\author{
Will it make a difference?
}

\author{
David Mabey professor, Stefan Flasche lecturer, W John Edmunds professor
}

London School of Hygiene and Tropical Medicine, London WC1E 7HT, UK

On 9 October the UK government announced that "enhanced screening" for Ebola virus disease will be implemented at Heathrow and Gatwick airports and Eurostar terminals. Details of how this will be done are not yet available, but the objectives presumably are to identify people arriving from Sierra Leone, Guinea, or Liberia who may have been exposed to Ebola, assess whether they have symptoms consistent with Ebola, test those who do, and isolate anyone with positive results.

Several practical difficulties will need to be overcome to achieve these objectives. As most direct flights to the UK from Sierra Leone, Guinea, and Liberia have been discontinued because of the epidemic, passengers will be arriving from various European cities, and itineraries will need to be carefully checked to identify passengers arriving from those countries. Those who are identified will be asked to complete a questionnaire stating whether they have been in contact with sick people or have attended funerals in west Africa, and whether they have symptoms such as fever, headache, diarrhoea, or vomiting. People who answer "yes" to any of these questions will presumably be referred to a health official, which is likely to lead to considerable delays; this would not be an incentive to fill in the form honestly. A thermal scanning device may also be used to check passengers' temperature on arrival, but it is unclear what will happen to those found to have a fever. Most will not have Ebola. Even if testing facilities are on site, substantial delays to large numbers of passengers seem inevitable, and isolation of all passengers waiting for their test results may prove challenging.

The World Health Organization recommends that passengers on international flights out of Sierra Leone, Guinea, and Liberia should be screened for evidence of Ebola before boarding their flight. Those with symptoms or a raised temperature should not be allowed on the flight. Clearly, identifying people with Ebola before they board an international flight is a desirable objective. But how well does this system work in practice? Data are not available on the number of passengers denied entry to a flight during the current epidemic, but there are strong incentives for those wishing to fly to deny symptoms even if they have them and to take an antipyretic such as aspirin to bring down their temperature if they have a fever.

\section{Lack of evidence}

Is there any evidence that screening travellers arriving at international airports is an effective way of identifying those with serious infections? The data from Canada, which introduced airport screening during the SARS (severe acute respiratory syndrome) epidemic, are not encouraging. A total of 677494 people arriving in Canada returned completed questionnaires, of whom 2478 answered "yes" to one or more question. A specially trained nurse referred each of these for in-depth questioning and temperature measurement; none of them had SARS. Thermal scanners were installed at six major airports. Of the 467870 people screened, 95 were referred to a nurse for further assessment. None of them was confirmed to have a raised temperature. The cost of this unsuccessful programme was \$CA17m (£9m; €12m; \$15m). ${ }^{1}$

Why was this measure so ineffective, and could it work now? During the SARS epidemic a simple model was used to assess the fraction of cases that could be detected by entrance screening. ${ }^{2}$ Assuming that people with symptoms are not allowed to board, entrance screening can only pick up those who develop symptoms while travelling. The longer the incubation period in relation to the flight duration, the lower the chance that this will happen, and the lower the yield from entrance screening. Updating the model using data on Ebola (incubation time 9.1 \pm 7.3 days $^{3}$; direct flight from Freetown to London 6.42 hours), we estimate that, if everyone with symptoms was denied boarding, about 7 out of 100 people infected with Ebola travelling to the UK would have symptoms on arrival and hence be detectable by entrance screening ( $95 \%$ confidence interval 3 to 13). The other $93 \%$ would enter the UK unimpeded. If passengers arriving via Paris or Brussels (journey time about 13 hours) were not screened in transit, entrance screening in the UK could detect up to $13 \%$ of infected people (95\% CI $7 \%$ to $21 \%$ ). The majority would still enter the UK before developing symptoms. Only if patients are allowed to fly irrespective of symptoms would entrance screening be able to detect a substantial fraction of cases ( $43 \%$ if there is no direct flight, 95\% CI $34 \%$ to $53 \%$ ).

People who know they are at risk and develop symptoms will want to seek care immediately, as they will fear for their lives. The priority should be to provide information to all those who may be at risk on how and where to seek care. This would be as effective as screening at a fraction of the cost.

Adopting the policy of "enhanced screening" gives a false sense of reassurance. Our simple calculations show that an entrance screening policy will have no meaningful effect on the risk of importing Ebola into the UK. Better use of the UK's resources would be to immediately scale-up our presence in west Africa-building new treatment centres at a rate that outstrips the epidemic, thereby averting a looming humanitarian crisis 
of frightening proportions. In so doing, we would not only help the people of these affected countries but also reduce the risk of importation to the UK.

Competing interests: We have read and understood BMJ policy on declaration of interests and have no relevant interests to declare.

Provenance and peer review: Commissioned; not externally peer reviewed.
1 St John RK, King A, de Jong D, Bodie-Collins M, Squires SG, Tam TW. Border screening for SARS. Emerg Infect Dis 2005;11:6-10.

2 Pitman RJ, Cooper BS, Trotter CL, Gay NJ, Edmunds WJ. Entry screening for severe acute respiratory syndrome (SARS) or influenza: policy evaluation. BMJ 2005;331:1242-3.

3 WHO Ebola Response Team. Ebola virus disease in west Africa-the first 9 months of the epidemic and forward projections. N Engl J Med 2014 Sep 22. [Epub ahead of print.]

Cite this as: BMJ 2014;349:96202

๑ BMJ Publishing Group Ltd 2014 